\title{
MISCELÁNEA NOTAS DE ENTONACIÓN GALLEGA
}

La entonación, según P. Denes, es la forma lingüistica en la que el hablante organiza determinadas informaciones ${ }^{1}$. Hay que partir, como aclara el mismo Denes en otro trabajo ${ }^{2}$, de los factores lingüísticos, $\mathrm{y}$, después, investigar cómo las características acústicas se relacionan con la entonación definida lingüisticamente y cómo algunas de esas rclaciones están influidas por otros factores.

Hasta ahora la entonación había sido definida únicamente como las variaciones de la frecuencia fundamental, o las variaciones de tono, pero las cosas se complican cuando se piensa que en un lenguaje cuchicheado, $\sin$ tono fundamental, también se percibe la entonación; luego éste no es su índice fundamental.

Las investigaciones realizadas por el mismo Denes en el artículo citado ${ }^{2}$ llegan a las siguientes conclusiones: a) la transmisión y la percepción de la información que lleva consigo la entonación se refleja a uivel acústico en un patrón complejo de índices de frecuencia fundamental, de intensidad $y$ de duración; b) también parece probable que la información que lleva consigo la entonación no sea codificada sólo en las dimensiones acústicas de frecuencia fundamental, de intensidad y de duración, sino que también la estructura de la frase y el contexto deben desempeñar su papel.

Tal combinación de factores hacen de la entonación un problema de los más complejos.

En español hay varios análisis de la entonación: el de Navarro Tomás ${ }^{3}$, que utiliza el análisis configuracional, y los de Stockwell,

1 P. DENES, A Preliminary Investigation of Certain Aspects of Intonation, en Language and Speech, 1959. II, p. ro6.

2 Further Studies in Intonation, en Language and Speech, 1962, V, p. I.

- Manual de entonación española, Nueva York, 1948. 
Bowen, Silva Fuenzalida ${ }^{1}$ y Bowen, Stockwell ${ }^{2}$, que emplean el análisis de niveles ${ }^{3}$. A nuestro modo de ver, todos son incompletos: el de Navarro está fundado sobre modelos literarios y su método es esencialmente fonético. Los restantes se limitan a estudiar patrones fundamentales, con el acierto de estar basados sobre el lenguaje coloquial, pero sin extenderse a ulteriores implicaciones en patrones más complejos, que evidentemente serian necesarios.

Sobre el resto de los dominios lingüísticos peninsulares, incluido el gallego, no existen trabajos en los que se haya aplicado una metodología empirica y acústica.

Para obtener los contornos melódicos que aquí mostramos, y muchos otros más que nos han servido de comprobación, hemos empleado el nuevo modelo Sona-Graph 606 IB con la incorporación del samplificador de escalas» $6076 \mathrm{C}$, que tiene como fin amplificar las zonas de frecuencia que deseemos. Con esta innovación, nos evitamos todas las engorrosas operaciones que debíamos utilizar anteriormente para la obtención de los contornos tonales 4.

Nosotros hemos utilizado en nuestro trabajo el análisis configuracional y el de niveles, montando éste sobre aquél, para no dejarnos llevar por la intuición o el subjetivismo, como ocurre en la mayoría de los análisis de niveles realizados por los lingüistas norteamericanos.

Utilizamos en este análisis tres niveles de tono, / $1,2,3 /$ y dos junturas terminales, $/ \bar{\psi}, \mathbf{k}:$ creemos que, al menos, en gallego, la juntura terminal de suspensión, $/ \rightarrow /$, cuando se da, es variante alojuntural de las otras dos ${ }^{5}$.

\section{r. Caracteristicas principales de la entonación gallega}

El rasgo principal que caracteriza la entonación gallega es un considerable aumento cuantitativo de la vocal sobre la que se manifiesta

\footnotetext{
I Spanish Junctucre and Intonation, en Languaje, 1956, XXXII, pp. 64I-665.

2 Patterns of Spanish Pronninciation: Chicago, I960. Hay aún otros dos trabajos inportantes que siguen de cerca los anteriormente señalados: DANIEL, $N$. CARDExas, Introducción a una comparación fonológica del español y del inglés, Washington, I960, y Josrirl F. MLArr,UCK, Entonación hispanica, en Anuario de Letras, Méjico, 1955. V.

3 Segrin DwitinT I. BoL,INGI:R, Intonation: Levels versus Configurations, en IVord, 195t, VII, ply. 199-210.

- Para mis detalles viase A. Quil,Is, Estructura del encabalgamiento en la mélrica espainola. Madricl, 1964, pp. 133-137.

s Jor dificultules de imprenta, no se consignan en la transcripción los fonemas medioabiertos y mediocerrados del sistema voćlico del gallego.
} 


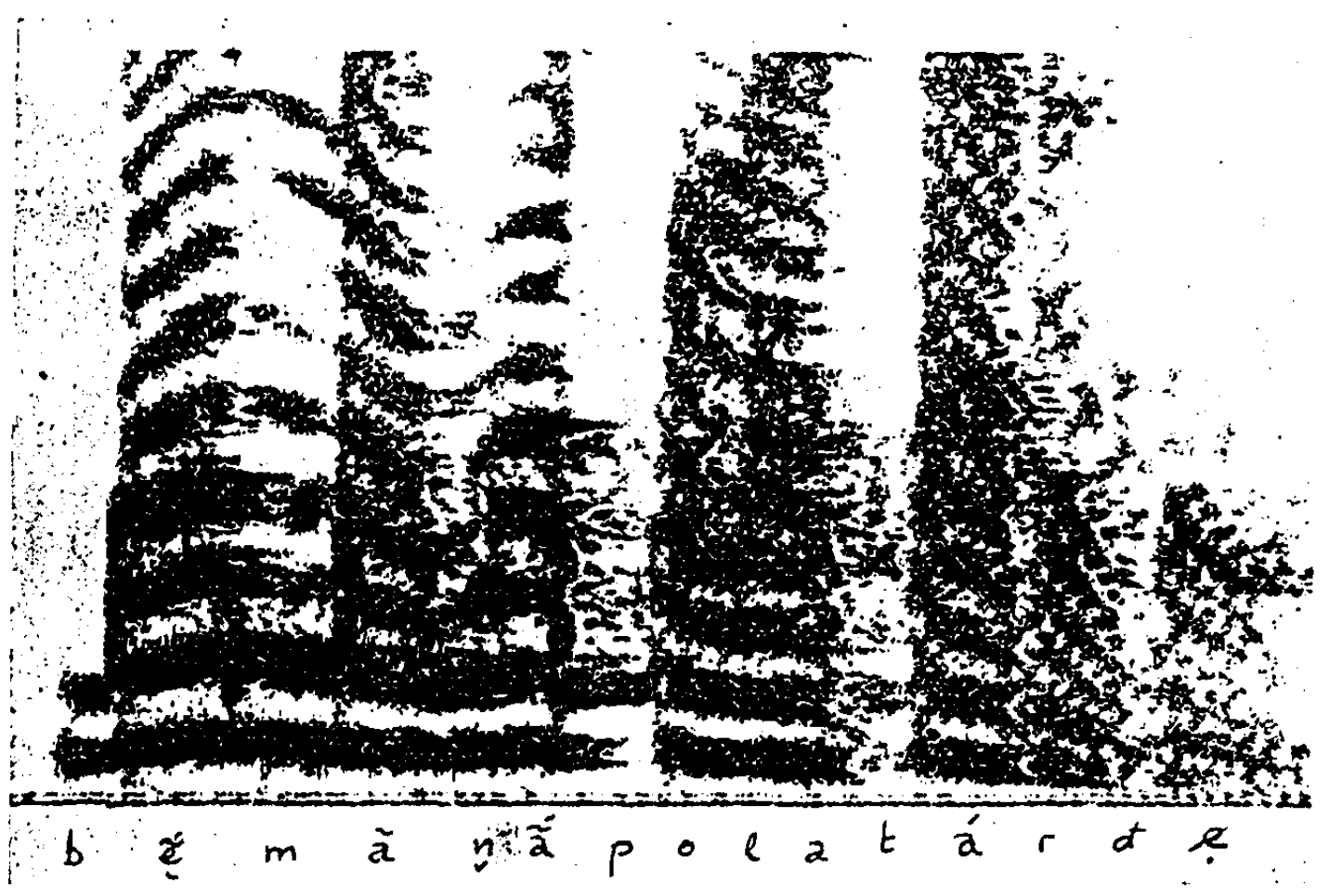

IESPIOTROXIRAMA I

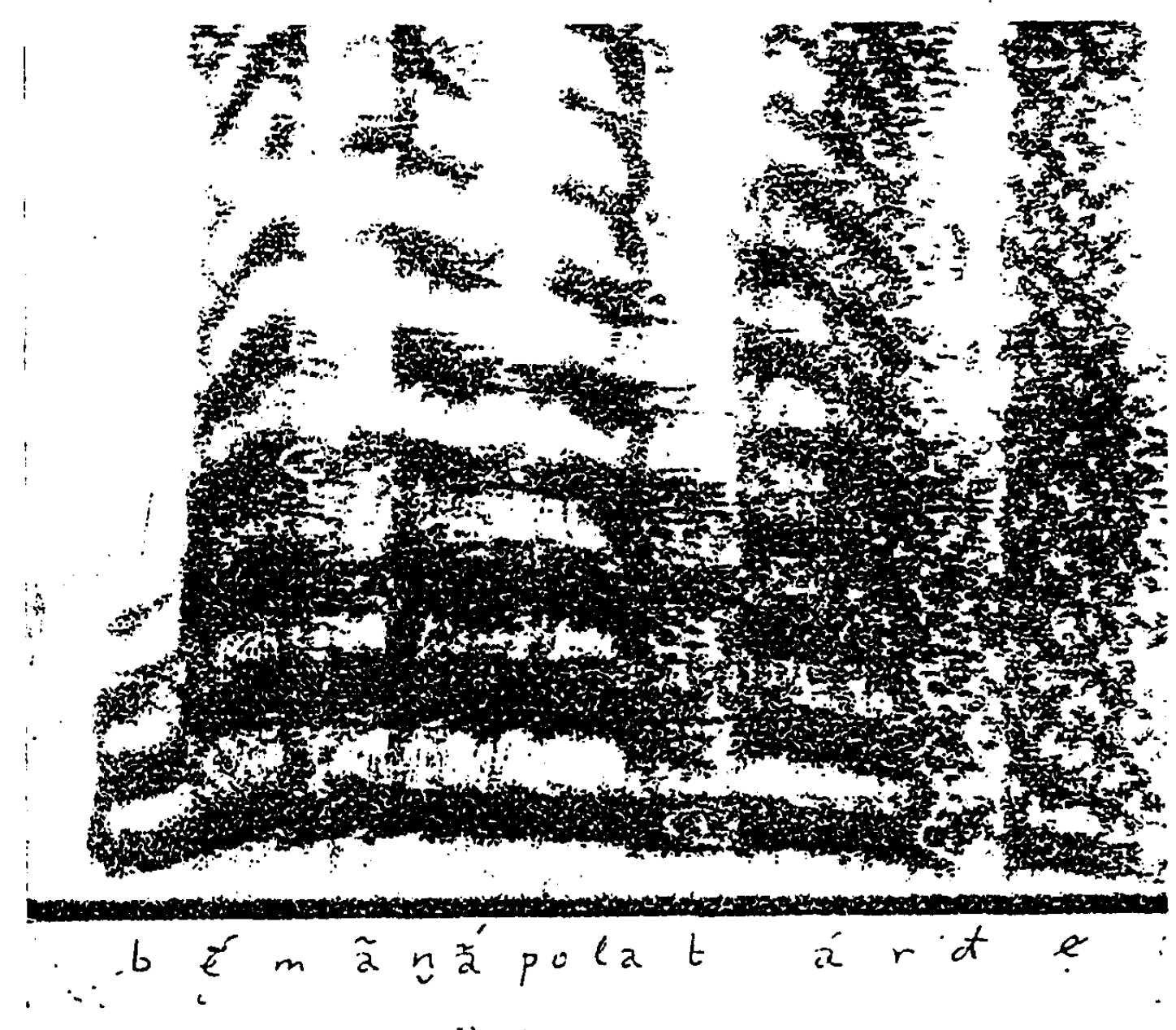

ISSPICTROGRAMA 2 


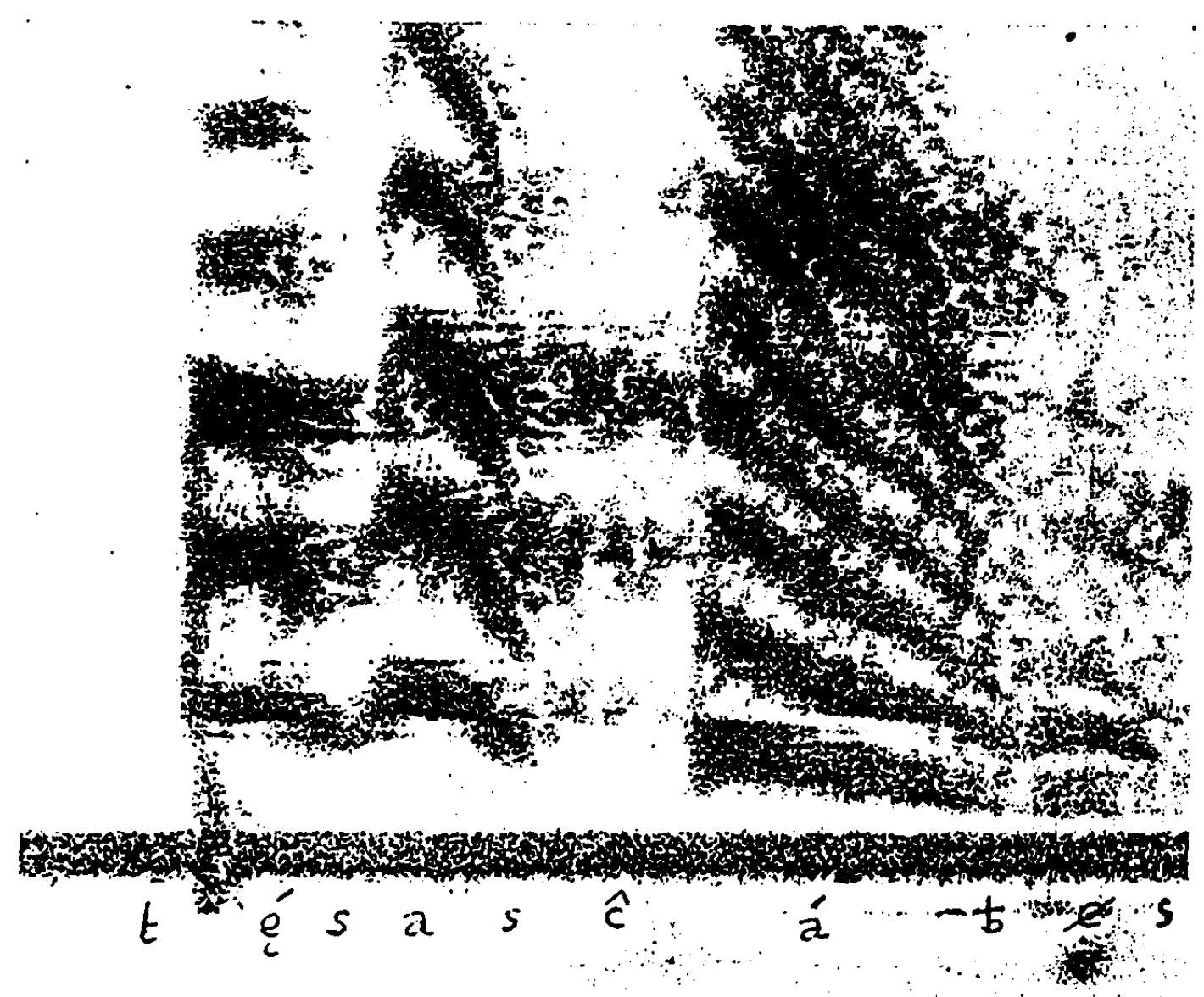

IESIPCTROGKAMI 3

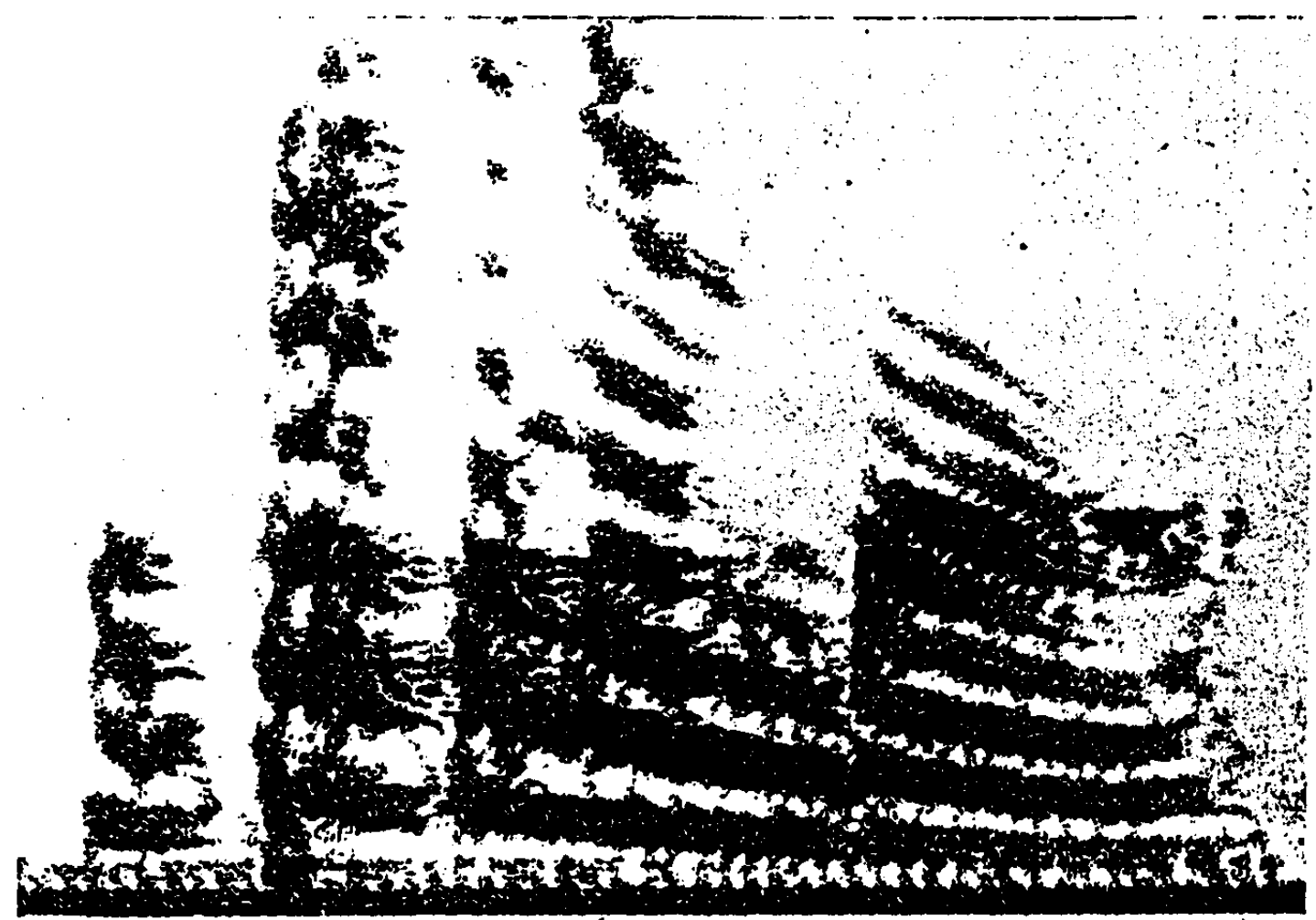

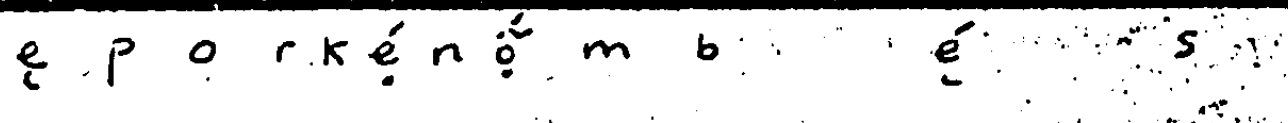

lisprectrograma 4 


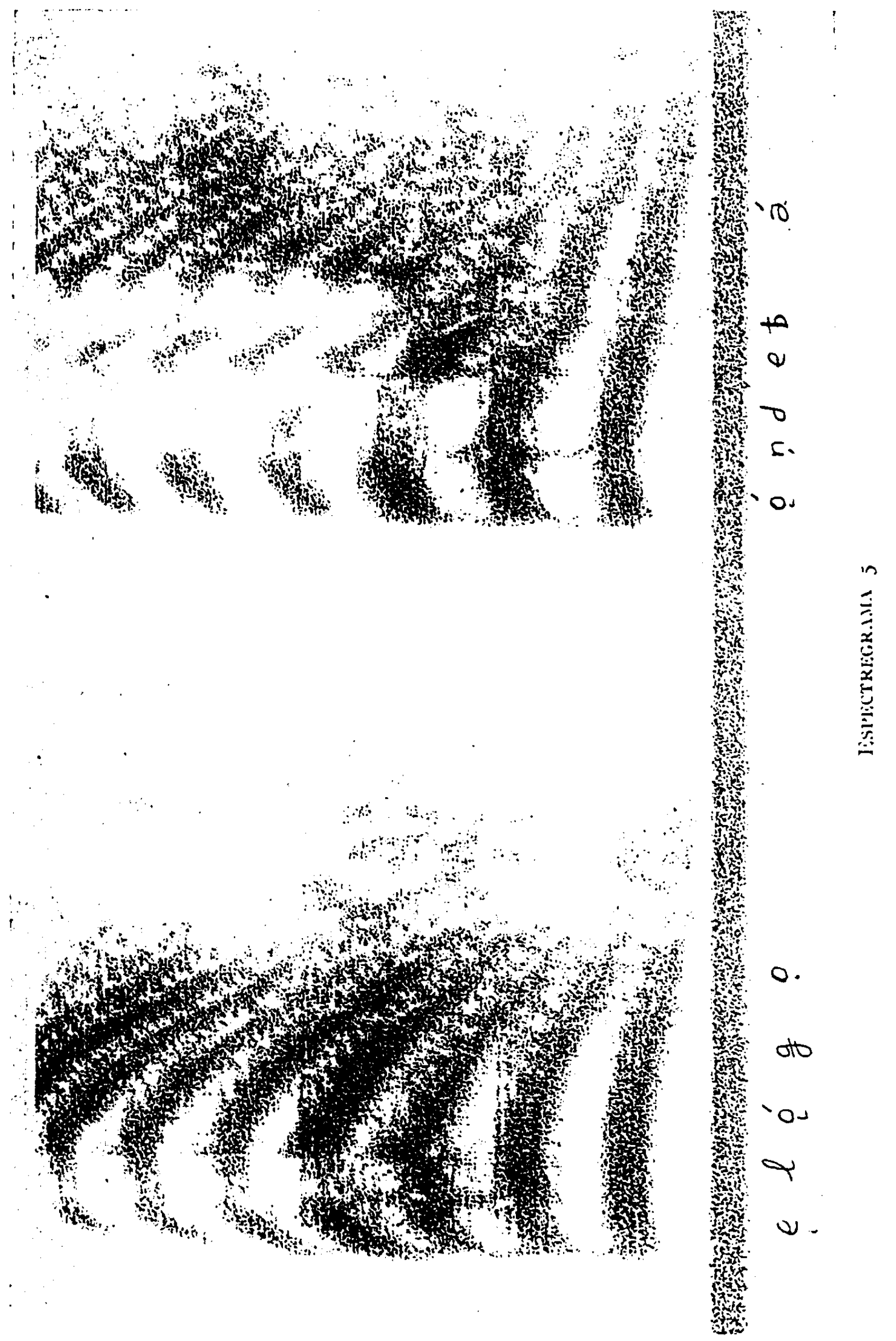




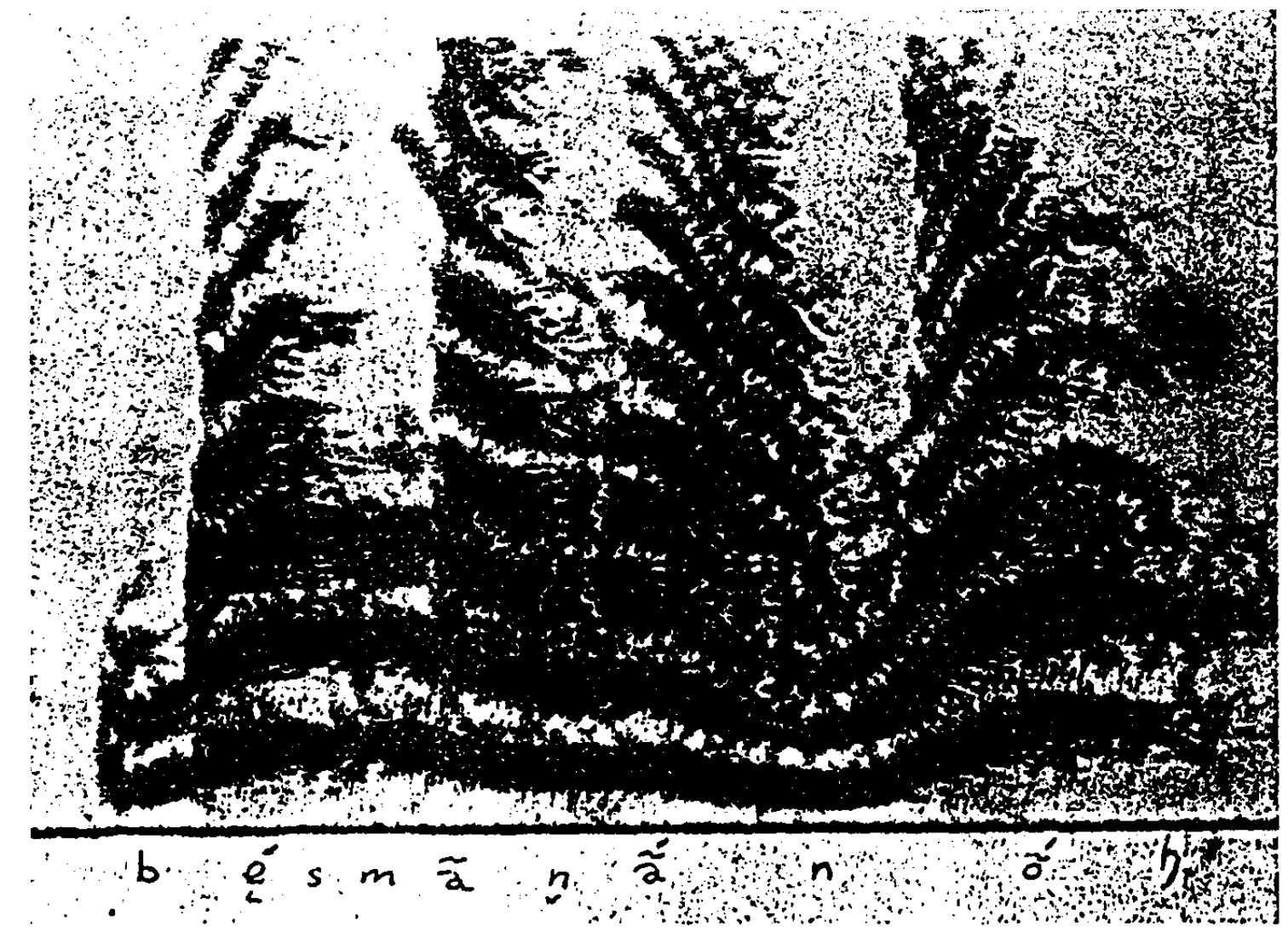

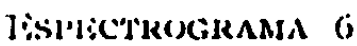

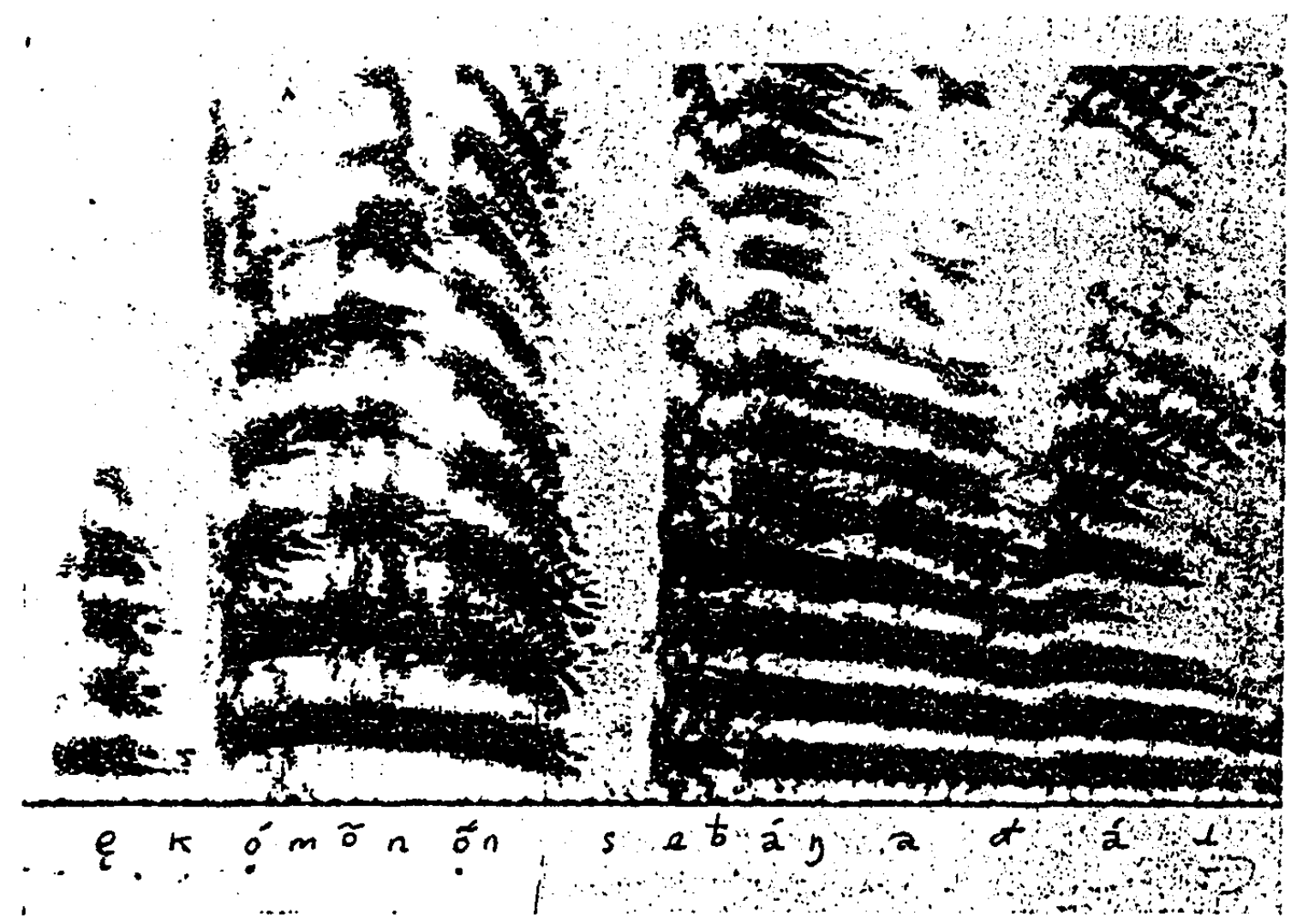

SEMTITHOCRAMA 7 


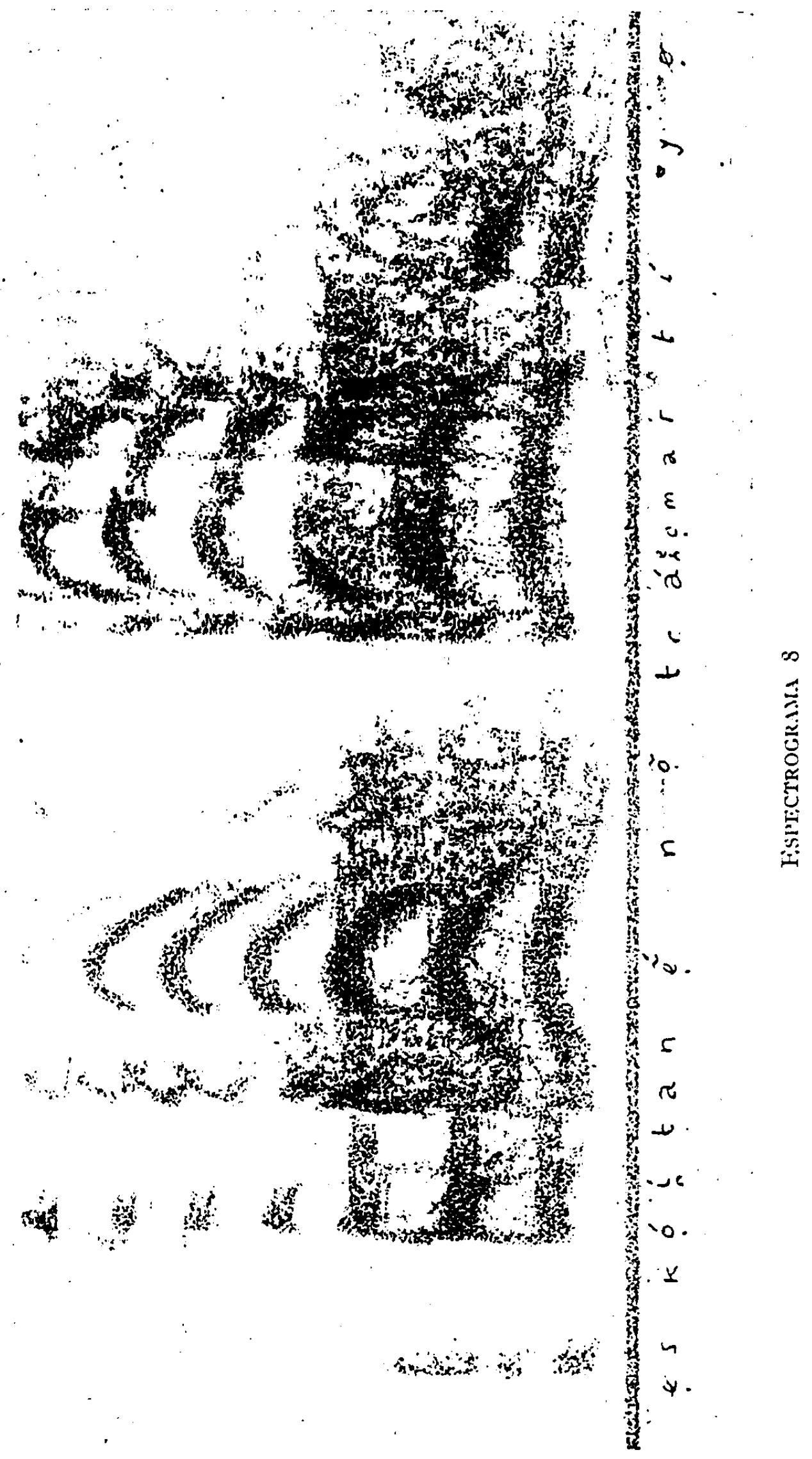




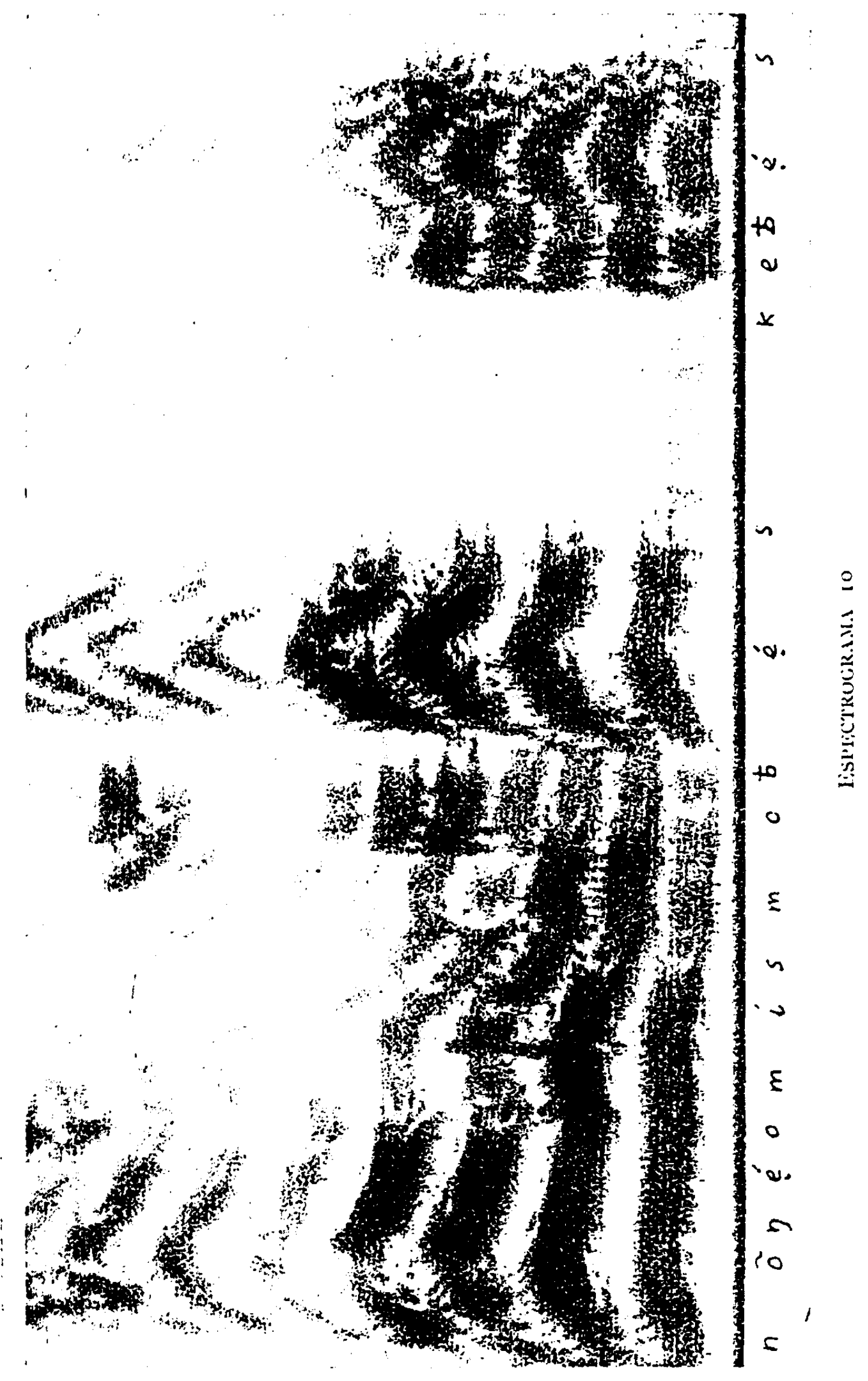


la inflexión tonal. Al producirse normalmente en el mismo segnento estos dos factores - mayor duración y variación de tono-infieren sobre él una puesta cn relieve peculiar, que es la que marca la característica más comúnmente conocicla de esta lengua.

\section{I.I. La enlonación en el ennuciado afirmativo}

La eutonación que acompaña a un enunciado afirmativo presenta una juntura terminal descendente, $/ \mid /$, y no tiene ningún otro elemento marcado en el interior del macrosegmento tonal. Obsérvese el espectrograma n. ${ }^{\circ}$ I. Su patrón tonal sería el siguiente:

\footnotetext{
$\begin{array}{lllllll}2 & \text { I } & 2 & \text { I } & \mathrm{I} & 2 & \text { I }\end{array}$

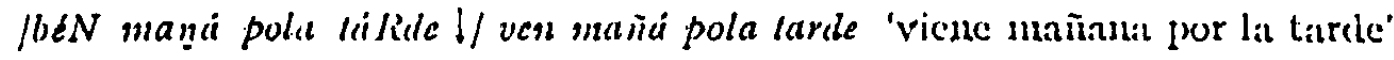

Iis lecir: /2 I 2 I I 2 I $1 /$

\section{r.2. La entonación en el ennuciado interrogativo}

La entonación, como se sabe, tiene la función de poder convertir un enunciado afirmativo en interrogativo. Esto es lo que se ha realizado en el entunciado del espectrograma n. ${ }^{\circ}$ 2: ha converticlo la afirmación del espectrograma n. ${ }^{\circ}$ I en interrogación. Al comparar estos dos espectrogranas, inmediatamente se percibe como distintivo una fuerte elevación de tono sobre la sílaba inicial. Esta elevación de tono y sólo ella es la responsable del rasgo de enunciado interrogativo. También se podría señalar como rasgo, si bien no pertinente, sí configurativo, el aumento de duración en la [á] de tarde, sobre la que también se produce el cambio de tono. La juntura terminal es descendente, al igual que en la afimación. Iil patrón tonal sería el siguiente:

$$
\begin{aligned}
& 322 \text { I I } 2 \text { I } \\
& \text { lbéN manci pola lálide! } 1
\end{aligned}
$$

Es decir: /322 I I 2 I //, donde lo distintivo con relación al patrón afirmativo son los dos primeros niveles de tono, $/ 32 /$.

E spectrograma 11.03 muestra otra frase interrogativa. La diferencia de ésta con la anteior reside en la seguridad de la respuesta que 
se espera. Mientras en el enunciado del espectrograma $110^{\circ} 2$ no se puede predecir la contestación, en el del n. ${ }^{\circ} 3$ se espera una contestación sí - 20 , pero prevista por el interlocutor que pregunta. La diferencia de niveles tonales en las dos primeras sílabas es notable con la parte final dei mismo enunciado $y$ con los segmentos situacionalmente análogos del espectrograma anterior. Su patrón tonal sería:

\section{$\begin{array}{llll}3 & 3 & 2 & 1\end{array}$ \\ |tés as cabes $\mid /$ ites as chaves? '¿tienes las llaves?"}

Es decir: / $332 \mathrm{I} \mathrm{I} /$. Juntura terminal descendente como en los anteriores, pero niveles de tono $/ 3$ /, iniciales, en lugar de /3 2/ del espectrograma anterior.

El espectrograma n.o 4 representa otro enunciado interrogativo introducido por el elenento interrogativo, por que. La juntura terminal es descendente, como lo es en este caso también en castellano. Fil rasgo pertinente de la interrogación se encuentra situado en el nivel tonal de la primera sílaba, donde se inicia la verdadera frase interrogativa: [por]. Su patrón tonal sería semejante al del espectrograma n. ${ }^{\circ}$ :

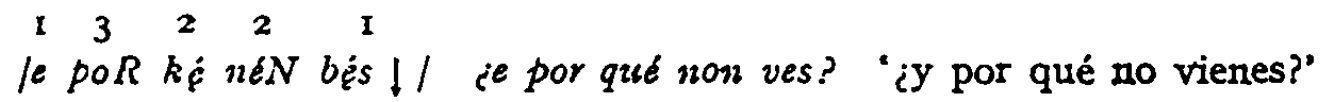

Es decir, / (I) $32221 /$.

También habría que señalar como peculiaridad el aumento de duración del segmento [é].

El espectrograma n. 5 muestra un enunciado interrogativo constituido por dos miembros: ambos presentan juntura terminal descendeute y elevación de tono en las primeras sflabas; sin embargo, una nayor elevación tonal presenta el miembro que conlleva todo el aspecto significativo, $y$ que es donde también reside el factor entonación al llevar el elemento interrogativo [ónde].

El patrón tonal de este enunciado es el siguiente:

$\begin{array}{llllll}3 & 2 & I & 3 & 2 & I\end{array}$

le logo | óNdeba $\mid /$ ¿e logo, ònde va? "¿y luego, adónde va?"

Es decir: /32 I 132 I $1 /$, patrón que conserva como peculiaridad de la forma interrogativa el nivel tonal $/ 3 /$. 


\section{r.3. La entonación en el enunciado interrogativo ratificado}

El espectrograma 11.06 es un enunciado interrogativo ratificado del mismo tipo de los que se forman en castellano añadiendo ¿no? o ¿vcrdad?

Iil cuerpo del enunciado [bésmãnáa presenta una juntura terminal descendente, como el resto de las interrogaciones. El nivel tonal de la primera sílaba es muy alto, siguiendo también el nuismo módulo de este tipo de enunciados.

La partícula negativa, gramaticalizada, presenta un movimiento de ascenso muy brusco, aunque después del núcleo silábico muestra una ligera tendencia al descenso, que no es perceptible.

El patrón tonal de este enunciado es el siguiente:

32 I 3

|bésmana! noฑ †/ ¿ves mañi, non? ¿vienes mañana, no?’

Lis decir: $/ 32$ I $131 \%$

Dejando a un lado el caso de la interrogación ratificada, señalada en el párrafo anterior, el gallego siempre utiliza juntura terminal descendente para la interrogación, sea del tipo que fuere; como por la juntura terninal coincide con la afirmación, se vale del recurso de elevar el tono por encima de su nivel normal para crear el rasgo que opone ambos tipos extremos de entonación. No hemos encontrado en nuestra investigación ningún tipo de juntura terminal ascendente como indice de enunciación interrogativa.

\section{I.4. La entonación en el enunciado exclamativo}

El espectrograna n. ${ }^{\circ} 7$ muestra un enunciado exclamativo. La juntura terminal es descendente, como en todos los demás casos, pero el descenso, en el tono de la parte final del enunciado, es mucho más lento que en la afirmación. Las primeras sílabas de la primera parte del enunciado, dejando a un lado el relator $\{e\}$, presentan un nivel de tono alto, similar al del enunciado interrogativo.

El patrón de este enunciado sería:

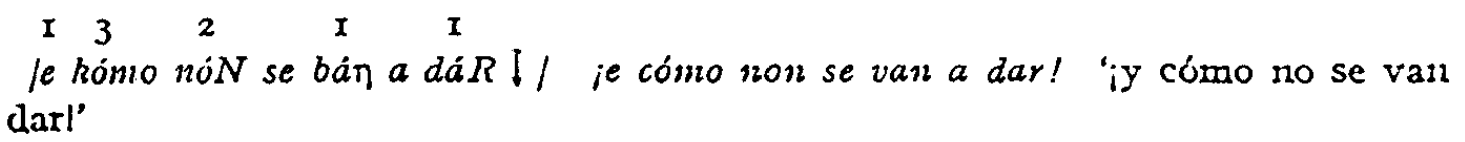

Iis decir: / (I) 32 I I $1 / \%$ 


\section{I.5. La entonación en el enunciado imperativo}

El espectrograma $11.0^{\circ} S$ representa dos enunciados: uno apelativo: [ẹkóịta nệnọ] y otro imperativo: [tráe o martiyọ], separados por una pausa. En el enunciado apelativo, la inflexión tonal está marcada en la silaba tónica del grupo sustantival neno. La juntura terminal es descendente.

El enunciado imperativo muestra también una juntura terminal descendente, pero lenta, similar a la del enunciado exclamativo. Las primeras silabas muestran un nivel tonal alto.

Los patrones de estos enunciados serían los siguientes:

I $\begin{array}{llllllll}2 & I & 3 & \text { I } & 3 & 2 & \text { I } & \text { I }\end{array}$

leskóita néno |/ träe o martilo |/ jescoita, neno, trae o martillo! 'irscucha, uiño, trae el martillo!'

Iis decir: / 2 I 3 I $1 / 32$ I I $1 /$.

\section{I.6. La entonación con un sintagma circunstancial hiperbatizado}

El espectrograma $110^{\circ} 9$ representa dos grupos fónicos con el sintagma circunstancial (temporal) hiperbatizado. Lo primero que se percibe ell el enunciado es la uniformidad de tono, que contrasta tremendamente con los espectrogramas anteriores. El comportamiento tonal es sinilar al del castellano: el sintagma circunstancial presenta una juntura terminal ascendente, y el sintagma verbal, descendente.

El patrón sería el siguiente:

$$
\text { I I } 23 \text { I } 2 \text { I I } 2 \text { I }
$$

|káNido salia \ eNtriba omanólo || canto salia, entraba o Manzolo 'cuando salia entraba (el) Manolo'

Is decir: / I I 231 I 2 I I 2 I $1 / 1 \%$

\subsection{La cnlonación en un ennnciado disynntivo}

Il espectrograma 11.0 Io representa un enunciado disyuntivo, que muestra los mismos morimientos tonales que en castellano: juntura terminal ascendente para el primer miembro y descendente para el segundo. 
El patrón sería: \\ /bés $\uparrow$ ke bés $\mid /$ (non é o mismo) vès que ves 110 es lo mismo vicnes \\ que ves'.
}

Ls decir: $/ 31$ I $21 /$.

\title{
2. Conclusion
}

Iil rasgo principal que caracteriza en general la entonación gallega es un considerable aumento cuantitativo de la rocal sobre la que se manifiesta la inflexión tonal. Estos dos elementos (aumento de duración y variación tonal) son los rasgos que contribuyen a la mencionada caracterización.

No hemos encontrado juntura terminal ascendente para la enunciación interrogativa. la juntura terminal descendente es constante en la enunciación afirmativa y en la interrogativa. I, o que distingue ambos enunciados es la aparición de un patrón tonal constituyente de nivel $/ 3 /$ en la primera sílaba de los enunciados interrogativos.

Sólo hemos encontrado juntura terminal ascendente en el segundo macrosegmento ('non?, ¿verdad?) de los enunciados interrogativos ratificados, fenómeno que también sc produce en castellano.

I.os enunciados exclanativos e imperativos aparecen con un nivel tonal /3/ en la primera sílaba, distinguiéndose su patrón tonal de los enunciados interrogativos en los niveles tonales finales del macrosegmento, que son / 2 I / para estos últimos, y / I I / en el exclannativo e impcrativo.

Los enunciados disyuntivos o hiperbatizados muestran el mismo comportamiento tonal que en castellano: juntura terminal ascendente después del primer macrosegmento, y descendente después del segundo.

RAMÓN B. CARRIL 